\title{
Research of The Harmful Gas Detection and Control System In Dairy
}

\author{
Yang Shifeng \\ College of Electronic information and automation \\ Tianjin University of Science \& Technology \\ Tianjin 300222, China \\ yangsf@tust.edu.cn
}

\author{
Liu Yajuan \\ College of Electronic information and automation \\ Tianjin University of Science \& Technology \\ Tianjin 300222, China \\ liuyajuan@mail.tust.cn
}

\begin{abstract}
This design constructs the harmful gas detection and automatic control system for NH3 and H2S in the dairy, which based on the electronic nose and LabVIEW virtual instrument technology development platform. This control system tests the harmful gas concentration of $\mathrm{NH} 3$ and $\mathrm{H} 2 \mathrm{~S}$ through the semiconductor gas sensor MQ-137 and MQ-136; The NH3 and H2S concentration change into the corresponding signals through the $A / D$ conversion chip, for the signal acquisition and processing; The system realize the functions of the harmful gas concentration of real-time data acquisition, display, storage, which used graphical programming language LabVIEW, and control the harmful gas concentration intelligently and automation. This control system has the advantages of high sensitivity, reliable work and the good effect
\end{abstract}

\section{Keywords- LabVIEW; Electronic nose; SCM; Gas detection}

Animal husbandry is an important part of the agricultural economy, and the breeding industry of milk cows is an important part of animal husbandry, the dairy not only provide in a large number of high-quality milk products but also provide the community with a large number of employment opportunities, improving the living quality, promoting the social economic development. It has played an important role for social progress. [1]

With the development of economy and the improvement of people's living standard, the demand of dairy products increasing promote the rapid development of the dairies. In recent years, the development of dairy industry is rapid, and the number of cows and milk products is total in the world, and industry development direction change into the scale, intensive and professional barn feeding level.

But it also appeared some problems. As is known to all, the modern animal husbandry production of four elements is variety, feed nutrition, environment management and disease prevention, the importance of livestock environment also gradually cause the attention of the authorities. These are two reasons, one is that the environment pollution by the cows excrement is more serious, becoming a new pollution source. If these excrement is not managed properly, it will affect the sustainable development of the breeding industry of milk cows, even threat to the human's health; The other is that a lot of peculiar smell harmful gas is produced by these waste, such as $\mathrm{NH} 3, \mathrm{H} 2 \mathrm{~S}$. These will hazard the cow health.

Dairy environment control is the key measures which should be meet the cow the best growth needs and improve the production level. Good control of the dairy is the guarantee to improve the environment dairy production capacity, get higher economic benefits and environmental benefits. Therefore, the harmful gas concentration is controlled effectively of the dairy, it is important significance for the growth of cows and milk production, give full play to its production potential and achieve high yield, high efficiency. [2]

This paper used electronic nose, LabVIEW and singlechip microcomputer technology, it design the dairy harmful gas effective control system, realized the dairy real-time data acquisition and automatic control, improve the work efficiency and reliability.

\section{THE SYSTEM STRUCTURE AND WORKING PRINCIPLE}

The LabVIEW virtual software platform developed upper computer control system, the system communicates through the RS-232 serial port and the next bit machine Atmega64 single-chip microcontroller, and the acquisition and conversion of data processing to Atmega64 microcontroller.[3]

Lower machine through the A/D conversion chip acquisition harmful gas gas concentration information, and transfer to PC; PC according to the acquisition of the current data and setting value judgement, down a machine a control information; Lower machine according to the PC control information, through the spraying facilities on milk field spray, and harmful gas, reducing harmful gas concentration, Make milk shop gas concentration less than set concentration, so as to achieve the purpose of control NiuChang gas concentration. System structure diagram 1 shows. 


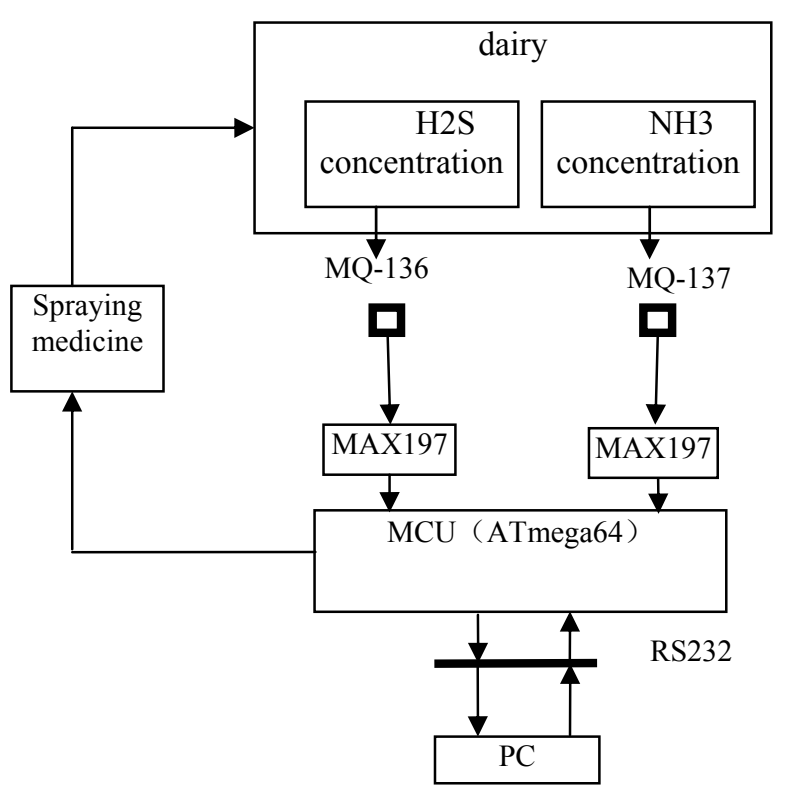

Figure 1. Structure of system

\section{HARDWARE DESIGN}

Hardware design includes: main controller, gas sensor, A/D conversion chip, serial communication, such as chip selection and interface circuit design.

\section{A. The main controller and interface circuit}

The main controller is using AVR series MCU ATmega64. ATmega64 one high performance and low power consumption of the eight microprocessors, working voltage of $4.5 \sim 5.5 \mathrm{~V}, 64 \mathrm{~KB}$ in the system of programmable Flash, $2 \mathrm{~KB}$ of EEPROM, $4 \mathrm{~KB}$ on chip SRAM, $64 \mathrm{~KB}$ optional external storage space, can through the SPI interface in the system of the programming. The main controller interface circuit as shown in figure 3 shows.(NH3 concentration is not more than $20 \mathrm{ppm}, \mathrm{H} 2 \mathrm{~S}$ concentration is not more than $8 \mathrm{ppm}$ )

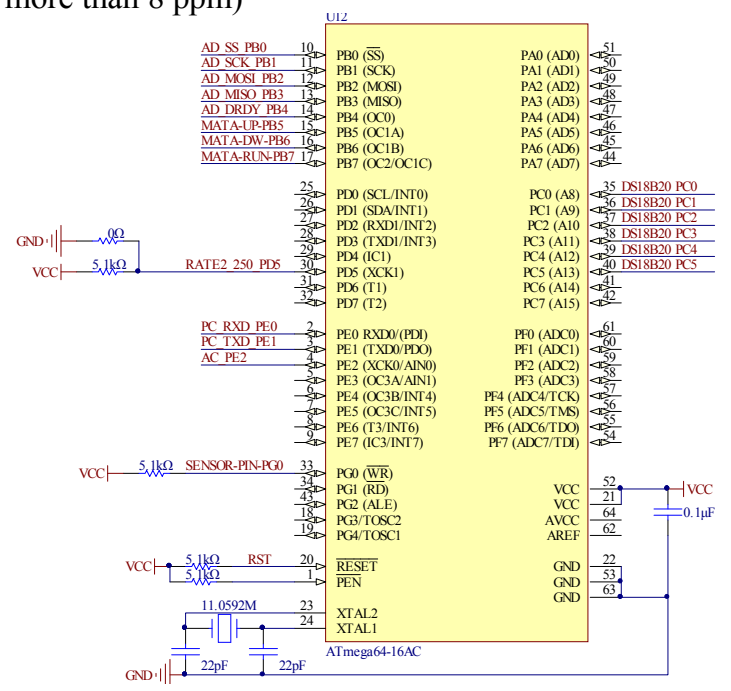

Figure 6. Schematic circuit diagram of host controller interface

\section{B. Gas sensitive sensor and $A / D$ conversion module}

System measuring ammonia and hydrogen sulfide of harmful gas concentration respectively with MQ-137 and MQ-136 semiconductor gas sensor. MQ-137 detection range for 10-300 PPM ammonia, MQ-136 detection range of 1-100 PPM hydrogen sulfide, which conform to the control system of concentration change requirements.

Packaging good gas sensor has only 6 needle tube feet, including four for signal out, 2 used to provide heating current. This kind of gas sensor for sensitivity adjustment is very important, so in order to ensure accurate measurement, ought to consider the influence of temperature and humidity field milk.

A/D chip as the core unit of data acquisition, data acquisition circuit design must according to the actual application carefully selection. In order to meet the accuracy of data acquisition, speed and channel parameters requirement, also in order to simplify the circuit design, we adopt the MAXIM company MAX197 as A/D conversion chip.[4]

MAX197 is one by using successive approximation way of working, the internal integration of the input tracking/hold circuit, Through the $8+4$ parallel interface and SCM connection, with two power down mode, through the software programming choose eight input channel a modulus conversion, Are widely used in industrial control system, robot, data acquisition system, medical instruments and telecommunication system. Used for gas concentration acquisition of gas sensors and A/D conversion interface circuit shown in figure 3 .

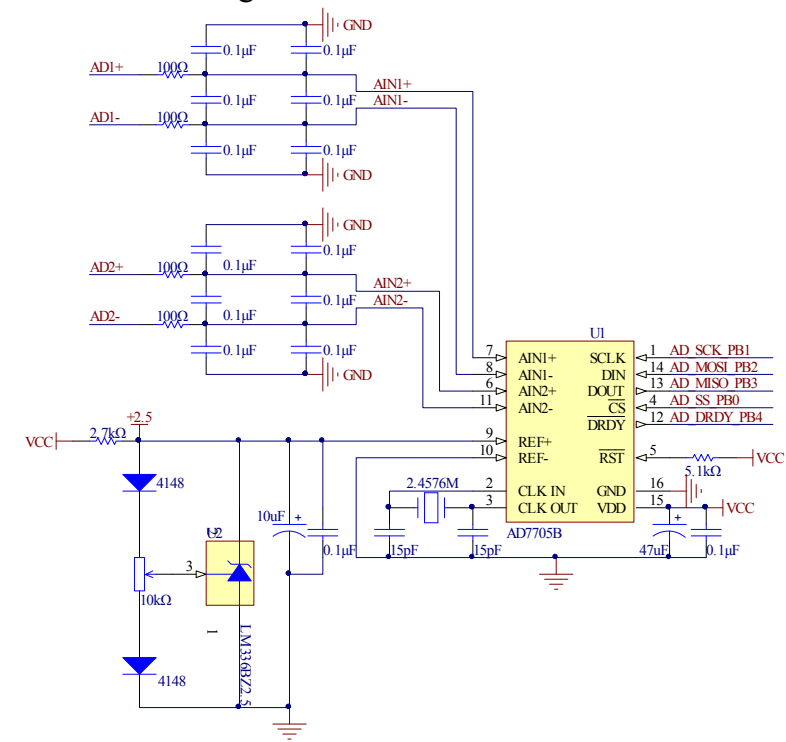

Figure 7. Schematic circuit diagram of gas sensor and A/D conversion

\section{Serial communication}

MAX232AESE chip is the RS-232 communication standard single power level conversion chip, the use of $+5 \mathrm{~V}$ power supply. MAX232 interface circuit as shown in figure 4.[5] 


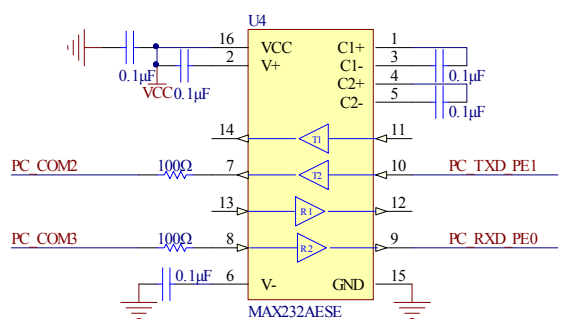

Figure 8. Schematic circuit diagram of interface of MAX232AESE

\section{SOFTWARE DESIGN}

\section{A. MCU programming}

The single chip microcomputer program written in $\mathrm{C}$ language, and its main finish the concentration of harmful gases, collection, storage and data sending work, and according to the PC control signal, control drug head open.

\section{B. PC program design}

Overriding the NI company LabVIEW as the development of information system software. LabVIEW development environment object oriented, and network, peripheral any integration, openness, high adaptability, short development cycle, be helpful for data processing, sharing and the deployment of simple, can reduce the development cost and time, reduce the cost of the whole monitoring system.

In the test and control system, the PC program design is the key link, mainly responsible for accept gas sensors to send data, through the writing of the corresponding program to receive data processing, to the production process monitoring, diagnosis and storage operation and related data analysis. LabVIEW provide hundreds of instrument source level driver, including DAQ, GPIB, VXI, RS a 232 and RS a 485 , according to the need can in LabVIEW developed by various hardware driver, also can through the dynamic link library (DLL) using other language development drive library, thus further expand its function.

In the test and control system, the PC through the harmful gas concentration control main program from lower machine gas concentration information processing, query and display, and a machine to a control command, and then control corresponding drug nozzle, complete dairy gas detection automatic control system.As shown in figure 5 .

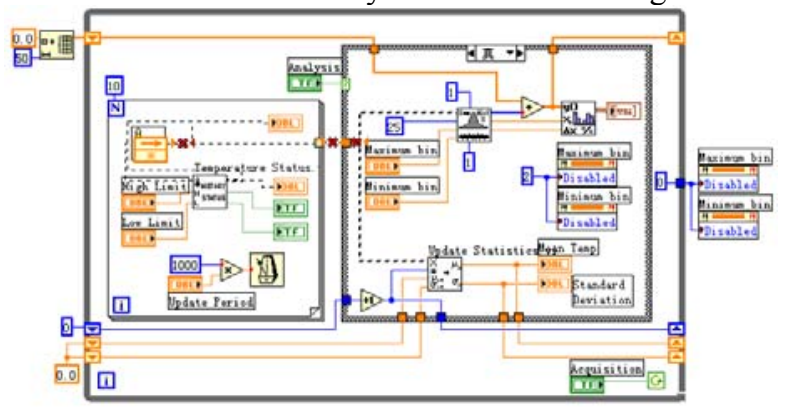

Figure 9. Data real-time acquisition module block diagram

\section{1) Gas concentration test procedures}

According to the literature, Design gas concentration measurement cycle program, a cyclic process is divided into the basic process: Acquisition and judgment to sprinkle medicine. The basic process is shown as shown in figure 6 .

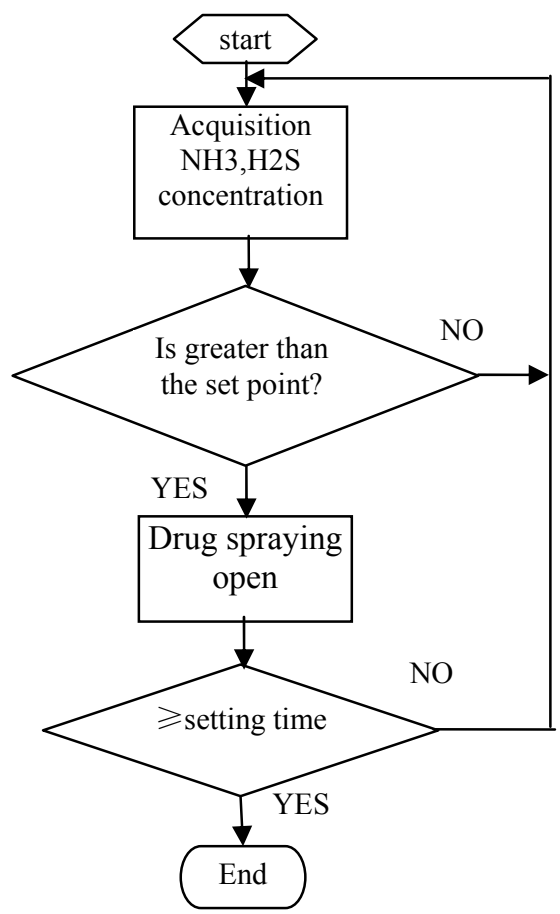

Figure 6. Basic flow chart

\section{2) Man-machine interface}

The man-machine interface including data display and system control model, the user can through the interface Settings gas concentration control parameters and inquires the history data, realize human-machine interaction.

In the system is running, can display the drug nozzle work and dairy air condition, etc. Under special circumstances, can by clicking on the drug head model to manual control spray drug shut, stop state.

\section{DESIGN RESULT}

The basic functions of information systems, including identification permissions, user management, recipe management, equipment operation, status monitoring, information recording and harmful gas concentration control.

\section{CONCLUSIONS}

This system use of the electronic nose technology and LabVIEW technology, realize the dairy harmful gas concentration of automatic control, with sensitivity and safety associated with adjustable, reliable, simple structure, long service life, low cost and easy to use. The results show that, the application of this system can control the milk shop harmful gas concentration, can be in small milk field use, can effectively improve the cows in the growth period of milk 
field environment, and reduce the labor intensity of their keepers.

\section{REFERENCES}

[1] Chen Xihui,Zhang Yinhong. 8.20 labview programming from entry to the master [M]. Beijing: Tsinghua University Press,2007

[2] Li Yuhua. Virtual instrument development platform LabVIEW software [J]. Computer Automated Measurement and Control, 1996(3) : 45-49.
[3] Zhongbai Zhou, Baoshan He. Multi-sensor technique and solid-state electrochemical sensor system for real-time and dynamic monitoring of multi-component gases[J]. Sensors and Aetuators B, 108(2005)379-384.

[4] Peter A.Blume . LabVIEW programming style [M]. Beijing: Publishing House of Electronics Industry, 2009: 198.

[5] Wang Shen, Liu Xuelan, Wang Yonghong. Research and implementation of environmental control automation system based on Chushi[J]. Computer Knowledge and Technology, 2008, (9): 17301732. 